\title{
PROTOKOLL DER 65. GENERALVERSAMMLUNG DER VEREINIGUNG ÖSTERREICHISCHER BIBLIOTHEKARINNEN UND BIBLIOTHEKARE
}

Datum: 18. November 2021, 16:00 Uhr

Ort: Universität Wien, Oskar Morgensternplatz 1, 1090 Wien

Vorsitz: Pamela Stückler/ Eva Ramminger

Protokoll: Markus Lackner

\section{TOP 1: Begrüßung und Feststellung der Beschlussfähigkeit}

Stückler begrüßt die Anwesenden und stellt die Beschlussfähigkeit fest.

TOP 2: Genehmigung des Protokolls der 64. Generalversammlung der VÖB

Es gibt keine Anmerkungen zum Protokoll der 64. Generalversammlung.

\section{TOP 3: Vorstellung des Bauer-Gedächtnispreises für Innovation}

Stückler: Die VÖB soll als Stifterin des Preises auftreten. Die Zielgruppe ist dabei auf Personen aus Österreich eingeschränkt. Preiswürdig sind Projekte, Publikationen und spezieller Einsatz im Bibliothekswesen. Die VÖB fördert mit EUR 1.000.-. Zusätzliches Sponsoring ist möglich. Der Preis ist in der neuen Geschäftsordnung verankert.

\section{TOP 4: Vorstellung Statuten neu}

\section{TOP 5: Vorstellung Geschäftsordnung neu}

Ramminger: Es gab mehrere Versionen der Statuten und der Geschäftsordnung. Die letzten Versionen wurden vor kurzem nochmals ausgesendet. Trotzdem gab es in der heutigen Vorstandssitzung noch kleine Änderungen, da Inkonsistenten festgestellt wurden.

Luzer: Der Auftrag Bruno Bauers war, die Statuten so schlank wie möglich zu machen und alles andere in eine Geschäftsordnung auszulagern. In der heutigen Vorstandssitzung hat Miehl noch gemeldet, dass die Wahl der vier Vorstandsmitglieder nicht abgedeckt war. Das wurde korrigiert. Präzisierungen gab es auch beim Stellenwert der Ehrenmitglieder. 


\section{TOP 6: Beschlussfassung über eingegangene Anträge}

\section{TOP 6a: Antrag auf Einrichtung des Bruno Bauer-Gedächtnispreises für Innovation}

Stückler stellt den Antrag auf Einrichtung des Bruno Bauer-Preises, wie in Tagesordnungspunkt 3 vorgestellt.

Der Antrag wurde einstimmig ohne Enthaltungen oder Gegenstimmen angenommen.

\section{TOP 6b: Antrag auf Genehmigung der Statuten}

Stückler stellt den Antrag auf Genehmigung der heute gezeigten Version der Statuten, mit der Möglichkeit, noch redaktionelle Änderungen durchzuführen.

Der Antrag wurde einstimmig ohne Enthaltungen oder Gegenstimmen angenommen.

\section{TOP 6c: Antrag auf Genehmigung der Geschäftsordnung}

Stückler stellt den Antrag auf Genehmigung der heute gezeigten Version der Geschäftsordnung, mit der Möglichkeit, noch redaktionelle Änderungen durchzuführen.

Der Antrag wurde einstimmig ohne Enthaltungen oder Gegenstimmen angenommen.

Stückler dankt Luzer für die Arbeit an der neuen Geschäftsordnung und den neuen Statuten.

Luzer macht darauf aufmerksam, dass ab diesem Zeitpunkt die neuen Statuten gelten.

\section{TOP 7: Wahl der Kassenrevisor/inn/en}

Stückler bittet um Handzeichen zur Zustimmung der Kassenrevisorlnnen Martin Kreinz und Ute Wödl.

Der Antrag wurde einstimmig mit einer Enthaltung angenommen.

Stückler bittet um Handzeichen zur Zustimmung der KassenrevisorInnenStellvertreterlnnen Wolfgang Hamedinger und Claudia Hausberger.

Der Antrag wurde einstimmig mit drei Enthaltung angenommen. Die Gewählten nehmen die Wahl an. 


\section{TOP 8: Allfälliges}

\section{TOP 8a: Beschlussfassung zur neuerlichen Verschiebung des 1. Österreichischen Bibliothekskongresses in Innsbruck}

Auf Beschluss des Vorstandes wird in der Generalversammlung der Antrag auf neuerliche Verschiebung des Bibliothekskongresses eingebracht:

Die Verschiebung wird damit begründet, dass Corona-bedingt die Stornierungsbedingungen (AGBs) des Congress Innsbruck so formuliert sind, dass die VÖB in der gegenwärtigen Situation alle finanziellen Risiken tragen müsste.

Ramminger stellt daher den Antrag, den Bibliothekskongress auf das Jahr 2024 zu verschieben, unter der Annahme, dass sich der BVÖ nur in geraden Jahren an einer gemeinsamen Veranstaltung beteiligen kann. Im Jahr 2022 soll aber trotzdem eine Interimsveranstaltung stattfinden.

Nach intensiver Diskussion über die Rahmenbedingungen der Abhaltung der Veranstaltung wird der Antrag mit einer Gegenstimme und drei Enthaltungen angenommen.

Schlacher stellt den Zusatzantrag, die Jahreszahl 2024 zu entfernen und „auf einen späteren Zeitpunkt" zu setzen. Der Antrag wird mit drei Gegenstimmen und drei Enthaltungen angenommen.

Feig/ stößt nun erst zur Generalversammlung dazu und stellt fest, dass der BVÖ auch bei einer Abhaltung 2023 mitmachen könnte und wird das in den BVÖ-Vorstand einbringen.

\section{TOP 9: Wahl der Präsidentin/des Präsidenten und ihrer/seiner Stellver- treter für die Funktionsperiode 2022-2023}

Der Vorsitz der Generalversammlung wird an Zemanek übergeben, während die zur Wahl stehenden Personen den Raum verlassen.

Zemanek bittet um Handzeichen für die Wahl des Präsidiumteams mit Seissl als Präsidentin, Ramminger als 1. Vizepräsidentin und Stückler als 2. Vizepräsidentin. Es wird einstimmig zugestimmt. Die Gewählten nehmen die Wahl an.

TOP 10: Wahl des Vorstandes der VÖB für die Funktionsperiode 20222023

Blumesberger gibt bekannt, ihre Kandidatur zurückzuziehen, da sie in der neuen Funktionsperiode die Tätigkeit der Schriftführerin übernehmen wird. 
Luzer verliest das Wahlergebnis:

1. Andreas Ferus

2. Christina Köstner-Pemsel

3. Marian Miehl

4. Markus Lackner

5. Marion Kaufer

6. Markus Stumpf

7. Ortwin Heim

Die ersten vier Personen sind somit in den Vorstand gewählt. Sie nehmen die Wahl an.

\section{TOP 11: Schlussworte der Präsidentin}

Seiss/ dankt allen für das Vertrauen, das die Präsidentin und die beiden Vizepräsidentinnen erhalten haben. Heuer wird noch eine Übergabesitzung und eine konstituierende Sitzung stattfinden. Es werden in der nächsten Funktionsperiode einige Themen bearbeitet, die heuer aufgrund der Situation nicht behandelt werden konnten. Ein weiterer Schwerpunkt des neuen Präsidiums ist der Ausbau des Weiterbildungsangebots. Die VÖB wird sich mit eigenem Vortragsprogramm im Rahmen von $4 \mathrm{~L}$ einbringen. Ziel ist es auch, Maßnahmen zu treffen, um das Zusammengehörigkeitsgefühl innerhalb der Bibliotheks-Community zu stärken.

DOI: https://doi.org/10.31263/voebm.v74i2.6552

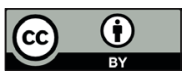

Dieses Werk ist - exkl. einzelner Logos und Abbildungen - lizenziert unter einer Creative-Commons-Lizenz Namensnennung 4.0 International-Lizenz 